\title{
Percutaneous Interventional Radiology-Operated Endoscopy for Foreign Body Removal
}

Mamdouh Khayat, MD¹; Adam Khayat ${ }^{2}$; Jeffrey Forris Beecham Chick, MD, MPH, DABR ${ }^{3}$; Travis L. Healey, MD ${ }^{4}$; Ravi N. Srinivasa, MD, FSIR 5

${ }^{1}$ Department of Radiology, Division of Vascular and Interventional Radiology, The Ohio State University Wexner Medical Center, 395 West $12^{\text {th }}$ Avenue, Columbus, OH 43210

2 Department of Biology, University of Louisville, 2211 South Brook Street, Louisville, KY 40208

${ }^{3}$ Division of Vascular and Interventional Radiology, Inova Alexandria Hospital

${ }^{4}$ Department of Radiology and Imaging Sciences, Indiana University School of Medicine

${ }^{5}$ Department of Radiology, Division of Vascular and Interventional Radiology, UCLA

Medical Center

All authors have read and contributed to this manuscript.

The authors have no releyant disclosures.

All individuals shown in the photographs have given their permission for inclusion in this manuscript and for publication.

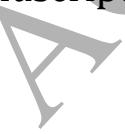

Correspondence/Reprints:

This is the author's manuscript of the article published in final edited form as:

Khayat, M., Khayat, A., Chick, J. F. B., Healey, T. L., \& Srinivasa, R. N. (2019). Percutaneous Interventional RadiologyOperated Endoscopy for Foreign Body Removal. Techniques in Vascular and Interventional Radiology.

https://doi.org/10.1053/j.tvir.2019.04.007 
Mamdouh Khayat, MD

Department of Radiology

Division of Vascular and Interventional Radiology

The Ohio State Wexner Medical Center

395 West $12^{\text {th }}$ Avenue

Columbus, $\mathrm{OH} 43210$

mamdouh.khayat@osumc.edu

ACKNOWLEDGEMENTS:

Special thanks to Anthony N. Hage, MD for graphical illustrations.

\section{ABSTRACT}

Image-guided retrieval of endovascular devices such as inferior vena cava filters, guidewires, and stents is well reported, though there is a paucity of published reports on biliary, genitourinary, or gastrointestinal foreign body retrieval utilizing percutaneous endoscopy, particularly when it is performed solely by interventional radiologists. In cases of failed traditional endoscopic techniques or to evade more invasive surgical options, percutaneous endoscopy can be an adjunctive tool employed by interventional radiologists to extract foreign bodies. In this article, clinical evaluation, perioperative management, and 
procedural techniques for biliary, genitourinary, and gastrointestinal endoscopy for foreign body retrieval are reviewed.

KEYWORDS: Percutaneous endoscopy; endoscopic retrieval; gastroscopy; endobronchial forceps; foreign body removal; interventional radiology

ABBREVIATIONS: ERCP - endoscopic retrograde cholangiopancreatography; EGD esophagogastroduodenoscopy 


\section{INTRODUCTION}

Percutaneous endoscopy utilized by interventional radiology has been sparsely documented in the literature [1-4]. Be it local interdisciplinary politics or inexperience with endoscopic tools, only a few interventional radiologists are employing percutaneous endoscopy despite its potential benefit in biliary, gastrointestinal, or genitourinary procedures.

Expanding from its use in the treatment of obstructive biliary pathology or as an adjunctive tool for nephrolithotomy, percutaneous endoscopy can also be effective in visualization and extraction of foreign bodies. Interventional radiologists are in a unique position to integrate percutaneous endoscopy as an adjunct to image-guidance for these difficult procedures.

\section{SETUP AND EQUIPMENT}

\section{Patient Selection}

Upon confirmation of the presence of a foreign body and prior to pursuing endoscopy-assisted extraction, a multi-disciplinary discussion with the referring physicians is necessary with inclusion of both medical and surgical subspecialists. This ensures that all parties agree with the proposed procedure and that surgical options are explored if endoscopic retrieval fails.

Pre-intervention laboratory evaluation often includes basic metabolic panel, complete blood count, and coagulation markers. Ideally, the international normalized radio should be less than 1.5 and platelet count should be greater than 50,000/ $\mu \mathrm{L}$. Given the potential of fluid shift during percutaneous endoscopy, electrolyte disturbances are corrected prior to pursuing endoscopy.

\section{Patient Setup and Obtaining Access}

Pre-intervention antibiotics should be prescribed based on the Society of Interventional Radiology guidelines. Procedures are typically performed under generalized anesthesia. Given the potential to infuse high volume fluids through the endoscope, orogastric and rectal tubes can be utilized to optimize fluid management and circumvent electrolyte disturbances.

Initial percutaneous access into the anatomical structure of interest is performed 
using standardized techniques. Ideally, this should be completed 4-6 weeks prior to scheduled endoscopy to ensure a mature transhepatic, transgastric, or transrenal tract.

\section{BILIARY ENDOSCOPY}

Intrabiliary foreign bodies, particularly dislodged or displaced stents, can often be retrieved by traditional endoscopic retrograde cholangiopancreatography (ERCP). However, in patients with altered anatomy, including prior hepaticojejunostomy or Billroth procedures, this can be difficult for obvious technical reasons. In this setting, percutaneous antegrade transbiliary endoscopy can be performed.

For choledochoscopy, a flexible endoscope is preferred to navigate through the angulations of the biliary tree. These could include a 9.5-Fr flexible disposable (Boston Scientific; Marlborough, MA), a 9-Fr flexible reusable (Olympus America; Center Valley, PA), or a 16.5- Fr flexible reusable (Olympus America) endoscope. Grasping forceps or snares can be advanced through the working channel of the endoscope to facilitate foreign body removal (Fig. 1, 2).

\section{GENITOURINARY ENDOSCOPY}

For genitourinary endoscopy, careful selection of the location of access is essential. This is dependent on the location of the foreign body- upper pole, lower pole, or both accesses may be needed. Prior to pursuing endoscopy, patients should be appropriately treated for any urinary tract infection. Tract dilation is performed using a high-pressure XForce balloon (Bard; New Providence, NJ). A peel-away sheath large enough to accommodate the chosen endoscope is inserted over the guidewire. In general, rigid endoscopy is preferred for upper tract foreign bodies and flexible endoscopy is preferred for more distal foreign bodies. The smallest caliber endoscope should be employed with an ideal working channel for the planned intervention. This decreases the potential of postprocedure urinary leakage. Following genitourinary endoscopy, a percutaneous nephrostomy catheter may be left in place. The nephrostomy tube is gradually downsized until it may eventually be removed.

\section{GASTROINTESTINAL ENDOSCOPY}

Once a percutaneous gastrostomy or gastrojejunostomy tract is established, the existing tract is dilated utilizing an 8-mm Bard X-Force (Bard Medical; Covington, GA) balloon. A 24-French Bard X-Force Teflon sheath is then often used through the dilated tract. For transgastrostomic endoscopy, a 22.5-French Olympus rigid endoscope (Olympus Corporation of America; Center Valley, PA) is preferred as this can be directed into the gastric lumen to visualize the foreign body. If more distal duodenal or jejunal visualization 
is required, a flexible endoscope can be considered (Fig. 3).

\section{TOOLS FOR FOREIGN BODY RETRIEVAL}

Once the percutaneous tract is established and a retained foreign body is fluoroscopically confirmed, direct visualization by endoscopy can be pursued to extract the foreign body. The tools selected for foreign body retrieval are dependent on the caliber of the working channel of the selected endoscope and the targeted foreign body to be retrieved. Traditional Amplatz Goose Neck Snares (Medtronic, Minneapolis, MN) can be readily used for retrieval. Alternatively, 3-mm endobronchial forceps with crocodile jaws (Lymol Medical; Woburn, MA) can be introduced through the working channel of rigid endoscopes to grasp stents or foreign bodies (Fig. 4,5). If the foreign body is small in caliber, ZeroTip Nitinol Stone Retrieval baskets (Boston Scientific; Marlborough, MA) can be considered and are available in 1.9-French or 3.0-French.

\section{DISCUSSION}

Though there may be initial trepidation of utilizing endoscopy, interventional radiologists are uniquely equipped to integrate percutaneous endoscopy as an adjunct to image-guidance for difficult procedures. Retrieval of endovascular devices such as inferior vena cava filters, guidewires, and stents has been well-reported [5-12]. However, there is a paucity of published reports on foreign body retrieval with combined endoscopy and image-guidance, particularly when it is performed solely by interventional radiologists.

The location of the foreign body presents unique challenges, often necessitating various approaches for endoscopy. In patients with surgically altered hepatobiliary anatomy not conducive to traditional ERCP, antegrade transbiliary endoscopy utilizing flexible or rigid endoscopes can be advantageous. This has been used previously in cases of stone extraction or recanalization of biliary strictures and these techniques can certainly be extrapolated and utilized for foreign body removal [1-4]. This also holds true for genitourinary endoscopy.

Transgastrostomic endoscopy has been utilized previously to evaluate upper gastrointestinal pathology and in the extraction of foreign bodies [13]. This can be helpful in cases of difficult traditional endoscopic evaluation, particularly in cases of persistent aspiration post-enteric tube placement or obstructive oropharyngeal tumors. Given the increased vulnerability of this patient population to complications from esophagogastroduodenoscopy (EGD), endoscopy through existing gastrostomy tracts is advantageous. Though it has been documented infrequently, it has proven to be safe for endoluminal evaluation of upper gastrointestinal pathology in the hands of gastroenterologists. Imaeda et al. reported a series of 43 patients who underwent 
transgastrostomic endoscopy with ultrathin endoscopes (5.5- $\mathrm{mm}$ and 6.5-mm) for evaluation of the upper gastrointestinal tract during routine exchange of gastrostomy tubes [14]. The use of transgastrostomic endoscopy can be expanded to the interventional removal of gastrointestinal foreign bodies.

Utilized as an adjunct to fluoroscopy, endoscopy by interventional radiology can be beneficial. Direct visualization of the target in three-dimensions allows for precise, purposeful movements without the need for continuous fluoroscopy, thus limiting radiation doses to the patient and the operator. Moreover, when utilizing devices such as endobronchial forceps in an organ, the use of combined fluoroscopy and endoscopy can potentially reduce the risk of injury when engaging the grasping mechanism. Directly visualizing the foreign body during capture may limit inadvertent injury, thus improving overall safety.

\section{CONCLUSION}

Interventional radiology operated endoscopy can be a valuable adjunct to help facilitate removal of foreign bodies without complications. Be it for biliary, genitourinary, or gastrointestinal indications, we speculate that it has the potential to reduce fluoroscopic exposure and inadvertent complications. 


\section{FIGURES:}

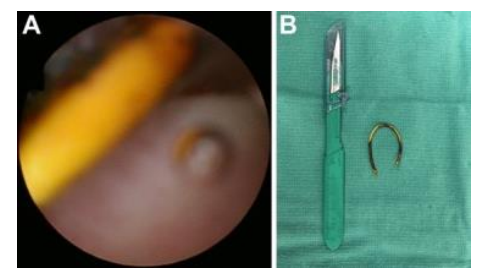

Figure 1: A, Retained plastic stent that had been present in the central biliary tree for over three years is seen under direct endoscopic visualization. This could not be removed previously despite numerous fluoroscopic attempts. B, Successful atraumatic removal using grasping forceps through a 16.5 French flexible endoscope.
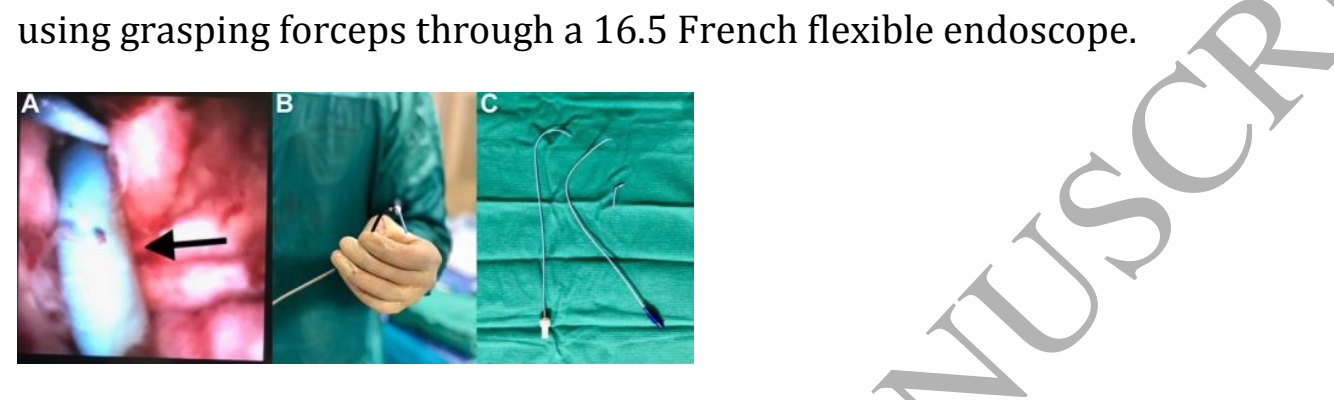

Figure 2: 52-year-old female with benign biliary stricture. A, Endoscopic image demonstrating fractured fragment from Accustick dilator set (arrow). B, C. Successful capture and removal of the fractured fragment using grasping forceps through a flexible endoscope.

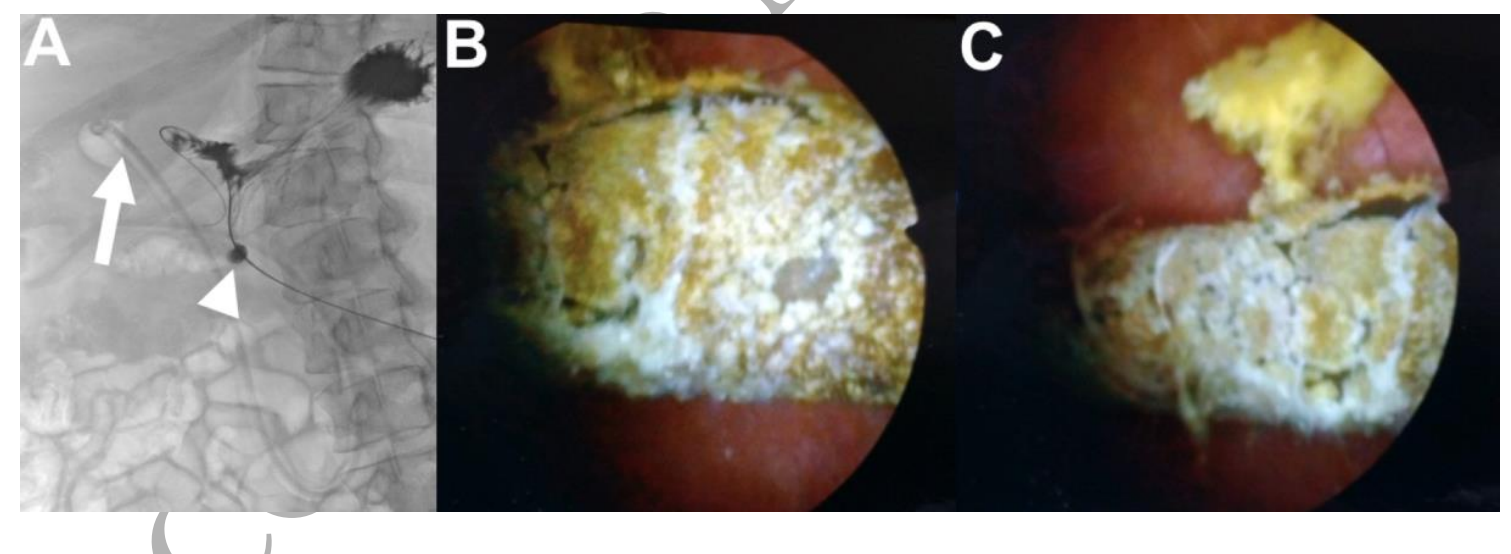

Figure 3: Fractured gastrojejunostomy tube with jejunal limb within the proximal small bowel. A, Fluoroscopic image demonstrating the deflated balloon of the gastric limb (arrowhead) and the fractured jejunal limb (arrow). Contrast is seen opacifying the stomach. B, C, Endoscopic images demonstrating the fractured jejunal limb within the proximal duodenum prior to removal 


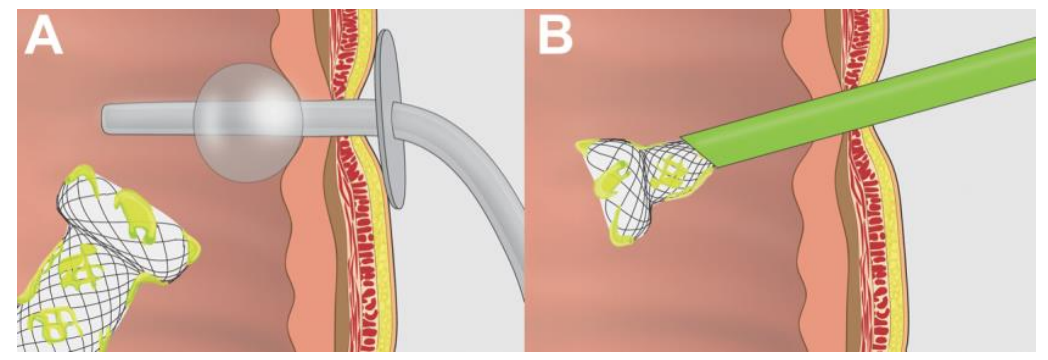

Figure 4: Diagrammatic illustration of Transgastrostomic Interventional RadiologyOperated Endoscopy and Foreign Body Removal. A, Retained foreign body is pictured in the gastric lumen. A gastrostomy tube is seen with the retention balloon inflated. B, Endobronchial forceps are used to retrieve the retained foreign body through a Teflon sheath under direct visualization.

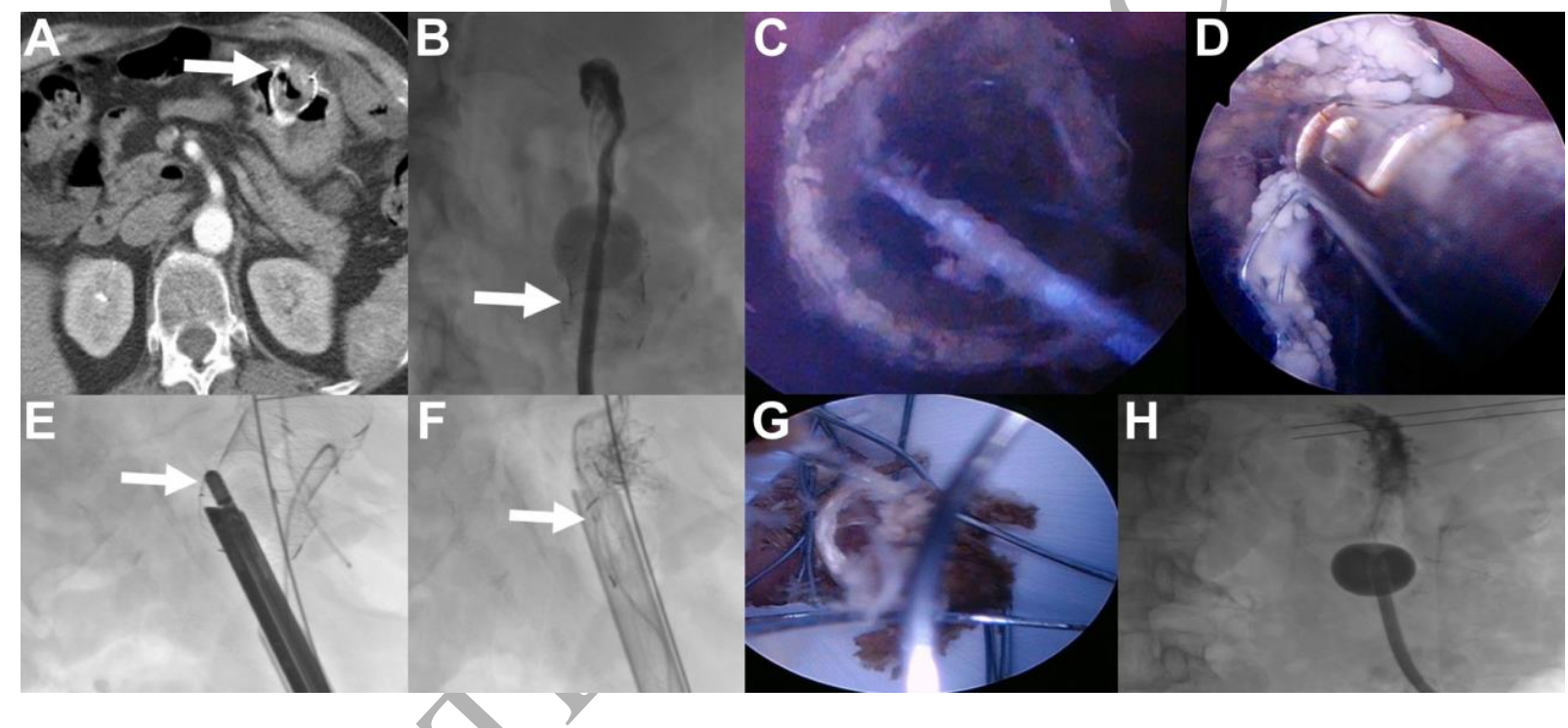

Figure 5: Gastric outlet obstruction status post pyloric stenting complicated by stent migration into wall of stomach and copious pericatheter leakage. A, Axial CT image demonstrating partial stent erosion (arrow) into the gastric wall. This explained the patient's significant leakage and pain. B, Fluoroscopic image demonstrating traction (arrow) on the stent after previous gastrostomy tube change. C, Endoscopic image demonstrating the stent floating within the stomach. D, Endoscopic image demonstrating endobronchial forceps grasping the stent through a rigid endoscope. E, Fluoroscopic image demonstrating successful capture of a portion of the stent (arrow). This was accomplished with a combination of endoscopy and fluoroscopy. F, Image demonstrating retraction of the stent into the cannula (arrow). G, Endoscopic image demonstrating partial retraction of the stent into the Teflon cannula. H, Final image demonstrating complete removal of the stent and replacement of gastrostomy tube within the stomach. The patient's clinical symptoms immediately improved. 


\section{REFERENCES}

1. Srinivasa RN, Chick JFB, Cooper K, et al. Interventional radiology-operated endoscopy as an adjunct to image-guided interventions. Curr Probl Diagn Radiol 2018; S0363-0188(18)30048-3.

2. Patel N, Chick JFB1, Gemmete JJ, et al. Interventional radiology-operated cholecystoscopy for the management of symptomatic cholelithiasis: Approach, technical success, safety, and clinical outcomes. Am J Roentgenol 2018; 210(5):1164-1171.

3. Patel N, Srinivasa RN, Gemmete JJ, et al. Disposable single-use choledochoscopy may facilitate recanalization of occlusive biliary anastomotic strictures. Radiol Case Rep 2017; 13(1):135-138.

4. Srinivasa RN, Patel N, Hage AN, et al. Interventional radiology-operated cholecystoscopy and cholecystolithotripsy: A guide for interventionalists. J Vasc Interv Radiol 2018; 29(4):585.

5. Shin BJ, Chick JFB, Stavropoulos SW. Inferior Vena Cava Filters: Placement and Retrieval. J Radiol Nurs 2015; 34:228-236.

6. Chick JFB, Stavropoulos SW, Shin BJ et al.A 16-F sheath with endobronchial forceps improves reported retrieval success of long-dwelling "closed cell" inferior vena cava filter designs. J Vasc Interv Radiol 2016; 27:1027-1033.

7. Chick JFB, Jo A, Meadows JM et al. Endovascular iliocaval stent reconstruction of inferior vena cava filter-associated iliocaval thrombosis: Approach, technical success, safety, and two-year outcomes in 120 patients.J Vasc Interv Radiol 2017;28:933-939.

8. Burke CT, Dixon RG, Stavas JM. Use of rigid bronchoscopic forceps in the difficult retrieval of the Gunther tulip inferior vena cava filter. J Vasc Interv Radiol 2007; 18:1319 1323.

9. Stavropoulos SW, Dixon RG, Burke CT, et al. Embedded inferior vena cava filter removal: use of endobronchial forceps. J Vasc Interv Radiol 2008; 19:1297-1301.

10. Kim YS, Lee SR. Percutaneous Forceps Retrieval of an Embolized Amplatzer Duct Occluder, Chonnam Med J. 2015 Aug;51(2):106-108.

11. Kukreja, K.U., Racadio, J.M., Alam, S. et al. Percutaneous retrieval of double-J stents using alligator forceps in children post renal transplant and pyeloplasty. Pediatr Radiol (2013) 43: 767. 
12. Kang JH, Rha SW et al. Successful Retrieval of a Fractured and Entrapped 0.035-Inch Terumo Wire in the Femoral Artery Using Biopsy Forceps. Korean Circ J. 2012 Mar;42(3):201-204.

13. Khayat M, Hussain JS, Chick JFB, Hage AN, Srinivasa RN. Percutaneous transgastrostomic interventional radiology-operated endoscopy facilitates foreign body removal using rigid endobronchial forceps. Diagn Interv Radiol 2018; 24: 42-45.

14. Imaeda $\mathrm{H}$, Hosoe $\mathrm{N}$, Nakamizo $\mathrm{H}$ et al. Clinical results of observation of the upper gastrointestinal tract by transgastrostomic endoscopy using an ultrathin endoscope. Journal of Gastroenterology and Hepatology 2010; 25: 1850-1854.

\section{COI STATEMENT}

The authors whose names are listed immediately below certify that they have NO affiliations with or involvement in any organization or entity with any financial interest (such as honoraria; educational grants; participation in speakers' bureaus; membership, employment, consultancies, stock ownership, or other equity interest; and expert testimony or patent-licensing arrangements), or non-financial interest (such as personal or professional relationships, affiliations, knowledge or beliefs) in the subject matter or materials discussed in this manuscript. 